\title{
The Effect of D-Galactosamine on Lean and Steatotic Rat Hepatocytes in Primary Culture
}

\author{
O. KUČERA ${ }^{1}$, H. LOTKOVÁ ${ }^{1}$, O. SOBOTKA ${ }^{1}$, Z. ČERVINKOVÁ $^{1}$ \\ ${ }^{1}$ Department of Physiology, Faculty of Medicine in Hradec Králové, Charles University in Prague, \\ Hradec Králové, Czech Republic
}

Received March 27, 2015

Accepted July 17, 2015

On-line December 15, 2015

\begin{abstract}
Summary
The aim of our work was to compare the effect of D-galactosamine (GalN) on primary cultures of lean and steatotic rat hepatocytes isolated from intact and fatty liver, respectively. GalN caused more severe injury to steatotic hepatocytes than to lean cells as documented by lactate dehydrogenase leakage. Necrotic mode of cell death strongly prevails over apoptosis since we did not observe any significant increase in activities of caspase 3, 8 and 9 in any group of hepatocytes treated with GalN. Reactive oxygen species (ROS) formation and lipid peroxidation were elevated in a dose-dependent manner by GaIN and were significantly more pronounced in fatty hepatocytes. A decrease in the percentage of hepatocytes with energized mitochondria was observed from $30 \mathrm{mM}$ and $10 \mathrm{mM}$ GalN in lean and steatotic hepatocytes, respectively. Our results undoubtedly indicate that steatotic hepatocytes exert higher sensitivity to the toxic effect of GalN. This sensitivity may be caused by more intensive GalN-induced ROS production and lipid peroxidation and by higher susceptibility of mitochondria to loss of mitochondrial membrane potential in steatotic hepatocytes. In our experimental arrangement, apoptosis does not seem to participate considerably on hepatotoxic action of GalN in either group of hepatocytes.
\end{abstract}

\section{Key words}

Fatty liver - D-galactosamine - Hepatocytes in vitro • Hepatotoxicity • Oxidative stress

\section{Corresponding author}

O. Kučera, Charles University in Prague, Faculty of Medicine in Hradec Králové, Department of Physiology, Šimkova 870, 50038 Hradec Králové, Czech Republic.

E-mail: kucerao@lfhk.cuni.cz

\section{Introduction}

Non-alcoholic fatty liver disease (NAFLD) belongs to the broad group of obesity-related disorders. It is characterized by the accumulation of lipid droplets in the liver which arises as a consequence of insulin resistance. NAFLD is believed to be the hepatic component of metabolic syndrome and it is the most common chronic liver disease in the Western world (Vernon et al. 2011). NAFLD is histologically defined by the presence of liver steatosis exceeding $5 \%$ of hepatocytes (Kleiner et al. 2005). In addition to simple steatosis, NAFLD includes non-alcoholic steatohepatitis (NASH) which can progress to fibrosis and finally to end-stage liver disease.

Recently, some authors found that NAFLD is associated with higher risk of drug liver injury when compared to general population (Nguyen et al. 2008, Tarantino et al. 2007, 2009). This may be caused by polypragmasia in patients with obesity, metabolic syndrome and NAFLD, and/or by liver alterations induced by NAFLD predisposing the liver to toxic injury (Fromenty 2013). These changes include altered expressions and activities of biotransformation enzymes (i.e. cytochrome P450 2E1) (Merrell and Cherrington 2011), mitochondrial dysfunction (Kucera et al. 2011a, Perez-Carreras et al. 2003), reduced amount of hepatic glutathione content, increased oxidative stress and lipid peroxidation (Garcia-Ruiz et al. 2006, Kucera et al. 2011a, Videla et al. 2004), augmented susceptibility of hepatocytes to apoptosis and necrosis (Bechmann et al. 2010, Feldstein et al. 2003) and cytokine/adipokine dysbalance (Tilg 2010). All mentioned factors contribute 
also significantly to the pathogenesis and/or progression of NAFLD.

In our previous experiments, we have proven that rat fatty hepatocytes show greater susceptibility to the toxic effect of acetaminophen (Kucera et al. 2012b) and thioacetamide (Kucera et al. 2011b) in vivo, and acetaminophen (Kucera et al. 2012a) and tert-butyl hydroperoxide (Garnol et al. 2014, Kučera et al. 2014) in vitro. Tert-butyhydroperoxide is a short-acting toxin causing relatively rapid damage by direct induction of oxidative stress in hepatocytes while acetaminophen for its toxic effect requires metabolic activation to reactive intermediate N-acetyl-p-benzoquinone imine. In comparison with tert-butyl hydroperoxide and acetaminophen, D-galactosamine (GalN) is a highly selective hepatotoxin with different mechanism of toxic action. GalN primary depletes the pool of uridine nucleotides in hepatocytes which inhibits transcription and protein synthesis in the liver (Keppler et al. 1970). This inhibition is consequently accompanied by increased oxidative and nitrosative stress, depletion of reduced glutathione and mitochondrial dysfunction (McMillan and Jollow 1992, Quintero et al. 2002, Ranchal et al. 2006). In vivo, GalN induces activation of innate immune response (i.e. Kupffer cells) by endotoxin (Stachlewitz et al. 1999) and strongly increases sensitivity of the liver to tumor necrosis factor $\alpha$ (TNF $\alpha)$ (Lehmann et al. 1987). GalN induces both necrosis and apoptosis in the liver (Cuesta et al. 2006, Kuhla et al. 2009) and in hepatocytes in primary culture (Siendones et al. 2005).

Considering different mechanism of GalN toxic action, the aim of our study was to find out whether steatotic rat hepatocytes in primary culture exert also higher susceptibility to GalN than lean hepatocytes, similarly as in the case of acetaminophen and tert-butylhydroperoxide. The emphasis of this work was given on a role of oxidative stress and a mode of cell death in GalN toxicity.

\section{Materials and Methods}

\section{Chemicals}

William's E medium (without phenol red), fetal bovine serum, penicillin, streptomycin and glutamine were purchased from Biochrom Ltd. (UK). Collagenase (Collagenase NB 4 Standard Grade from Clostridium histolyticum) was obtained from SERVA Electrophoresis GmbH (Germany), insulin (Actrapid, Hoechst, Germany) and glucagon (Novo Nordisk, Denmark) were from the suppliers mentioned in brackets. D-galactosamine, type I collagen, trypan blue, dexamethasone and all other chemicals were, unless otherwise stated, of at least analytical grade and obtained from Sigma-Aldrich (WI, USA).

Animals

Male albino Wistar rats (Velaz, Czech Republic) were housed at $23 \pm 1{ }^{\circ} \mathrm{C}, 55 \pm 10 \%$ relative humidity, air exchange 12-14 times/h, and 12-h light-dark cycle periods $(6: 00 \mathrm{~h}$ to $18: 00 \mathrm{~h})$. Rats used for isolation of non-steatotic hepatocytes were fed ad libitum a standard pelleted diet (Velas, Czech Republic; $10 \%$ energy fat, $30 \%$ energy proteins, $60 \%$ energy saccharides) for 6 weeks. For isolation of fatty hepatocytes, animals were fed a high-fat diet with $71 \%$ of energy derived from fat (lard), $18 \%$ from proteins and $11 \%$ from saccharides for 6 weeks. The animals had free access to tap water. All animals received care according to the guidelines set by the Animal-Welfare Body of Charles University in Prague, Faculty of Medicine in Hradec Králové, Czech Republic, and the European Guidelines on Laboratory Animal Care. All animal experiments were approved by the committee mentioned above and by the Ministry of Education, Youth, and Sports of the Czech Republic (authorization reference number 1315-28572/2012-30).

\section{Hepatocyte isolation, cultivation and treatment}

Hepatocytes were isolated from rat livers by two-step collagenase perfusion (Berry et al. 1991). The viability of freshly isolated hepatocytes used in experiments was more than $90 \%$ as confirmed by trypan blue exclusion test. Isolated hepatocytes were suspended in William's E medium supplemented with fetal bovine serum (6\%), glutamine, antibiotics and hormones (Kucera et al. 2012a) and plated in collagen-coated 12 -well plates $\left(4 \times 10^{5}\right.$ cells in $400 \mu 1 /$ well, NUNC) and 96-well plates $\left(3 \times 10^{4}\right.$ cells in $100 \mu 1 /$ well, NUNC).

Hepatocytes were allowed to establish monolayer in a humidified atmosphere containing $95 \%$ air and $5 \% \mathrm{CO}_{2}$ at $37{ }^{\circ} \mathrm{C}$ for $2 \mathrm{~h}$. Then the medium was replaced with a fresh supplemented medium without fetal bovine serum containing dissolved GalN $(0-40 \mathrm{mmol} / \mathrm{l})$. Based on our previous results, we have chosen treatment period of $24 \mathrm{~h}$. Then the medium was collected and cells were harvested for the required assays.

\section{Biochemical assays}

Hepatocyte injury was determined by lactate 
dehydrogenase (LDH) leakage. LDH activity in the culture medium and in cell lysate (Cell Lysis Buffer, Cell Signalling Technology, MA, USA) was measured using a commercial kit from DiaSys (Germany). The production of reactive oxygen species (ROS) was evaluated using 5and 6-chloromethyl-2',7'-dichlorodihydrofluorescein diacetate (CM-H2DCFDA; Molecular Probes, USA) as previously described (Kučera et al. 2014). Secondary end product of lipoperoxidation malondialdehyde (MDA) in culture medium was determined by the assessment of thiobarbituric acid reactive substances (TBARS) (Ohkawa et al. 1979). For better comparison of results from different experiments, values of ROS production and MDA concentration are expressed as percent of control non-steatotic hepatocytes $(100 \%)$.

Concentration of tumor necrosis factor $\alpha(\mathrm{TNF} \alpha)$ in culture medium was measured by enzyme linked immunosorbent assay (ELISA) according to manufacturer's instructions (BMS622, Bender MedSystems, Austria). Results are expressed as percent of control non-steatotic hepatocytes (100\%).

The activities of caspases 3,8 and 9 were assessed in cell lysate (Cell Lysis Buffer) after addition of specific substrates Ac-DEVD-AMC, Ac-LETD-AFC and Ac-LEHD-AMC, respectively (Enzo Life Sciences, NY, USA). The activities were measured in a fluorescent mode using a TECAN Infinite M200 spectrofluorometer (Tecan Group AG, Switzerland). The excitation and emission wavelengths were 350 and $460 \mathrm{~nm}$ for caspase 3 and caspase 9, and 400 and $505 \mathrm{~nm}$ for caspase 8. Results were normalized to protein concentration (Bradford 1976) and expressed as percent of control non-steatotic hepatocytes $(100 \%)$.

\section{Fluorescent microscopy}

Mitochondrial membrane potential (MMP) was depicted by fluorescence microscopy using JC-1 (Molecular Probes, Inc., Oregon, USA) as previously described (Kučera et al. 2014). Results are expressed as percentage of cells with energized mitochondria from all attached cells.

\section{Statistical analysis}

Results are expressed as means $\pm \mathrm{SD}$ of at least three independent experiments; $\mathrm{p}<0.05$ was set as the border for statistical significance. Statistical evaluation was performed using GraphPad Prism software 6.01 (La Jolla, CA, USA). Data were first tested for normality by means of D'Agostino \& Pearson omnibus test. All data did not follow Gaussian distribution, thus the data were analyzed by a non-parametric Kruskal-Wallis test followed by Dunn's multiple comparisons test.

\section{Results}

Figure 1A shows LDH leakage from lean and steatotic hepatocytes after 24-h incubation with GalN. In non-fatty cells, a significant increase in LDH leakage was observed from $30 \mathrm{mM}$ concentration of GalN $(\mathrm{p}<0.001)$. In steatotic hepatocytes, $\mathrm{LDH}$ leakage was elevated from $20 \mathrm{mM}$ concentration of GalN $(\mathrm{p}<0.05)$. We did not observe any increase in caspase 3, 8 and 9 activities in cell lysate in any group treated with GalN when compared with an appropriate control (Figs 1B, 1C and 1D).

ROS production was elevated by incubation with GalN in a dose dependent manner in both lean and steatotic hepatocytes (Fig. 2A). A significant increase in generation of ROS was found from 10 and $5 \mathrm{mM}$ concentrations of GalN in non-steatotic $(p<0.05)$ and fatty cells $(p<0.001)$, respectively. In steatotic hepatocytes, ROS production was significantly higher than in lean cells (5, 10 and $20 \mathrm{mM}$ GalN). MDA concentration in cell lysate is depicted in Figure 2B. Production of TBARS is dose-dependent. GalN at concentrations from $30 \mathrm{mmol} / 1$ induced a significant elevation of TBARS in steatotic hepatocytes, whereas only the highest tested dose of GalN (40 mmol/l) caused a significant MDA increase in non-fatty cells.

Figure 3 depicts concentration of TNFa in culture medium after $24 \mathrm{~h}$. Cultivation of hepatocytes with GalN led to a dose dependent increase in TNF $\alpha$ levels. Significant elevations of $\mathrm{TNF} \alpha$ levels were observed similarly from $20 \mathrm{mM}$ GalN in both lean and fatty hepatocytes.

In lean hepatocytes, GalN from concentration of $30 \mathrm{mmol} / \mathrm{l} \quad(\mathrm{p}<0.05)$ induced a significant decrease in percentage of cells containing energized mitochondria (Fig. 4). In contrast to this finding, steatotic hepatocytes exerted a significant reduction in the percentage from $10 \mathrm{mM}$ GalN $(\mathrm{p}<0.05)$. GalN at concentrations from 10 to $40 \mathrm{mmol} / \mathrm{l}$ induced more severe decrease in the percentage of hepatocytes with energized mitochondria in fatty cells. Figure 5 shows representative fluorescent microphotographs of lean and steatotic hepatocytes incubated without GalN or with $20 \mathrm{mM}$ GalN. 
A

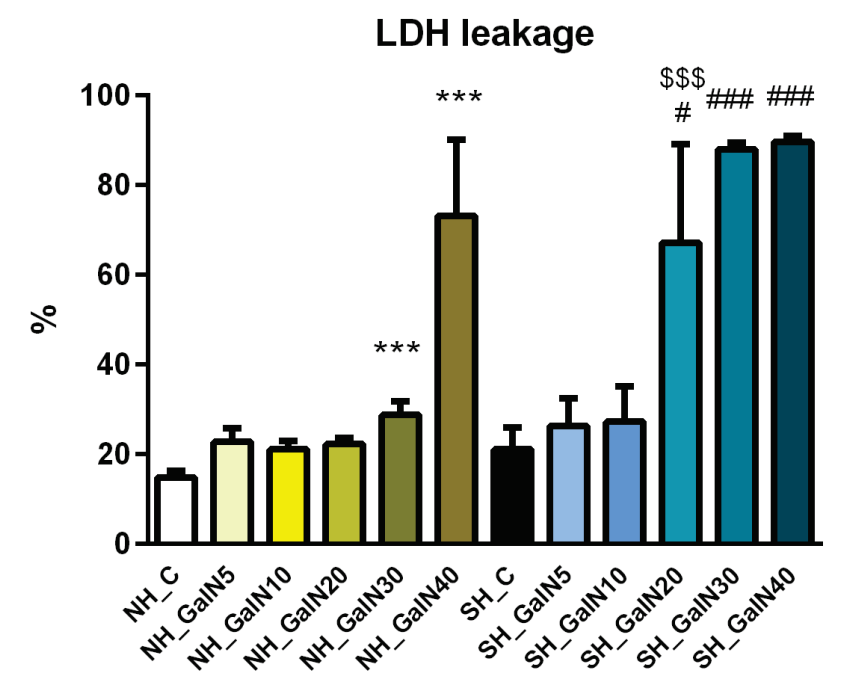

C

Caspase-8 activity in cell lysate

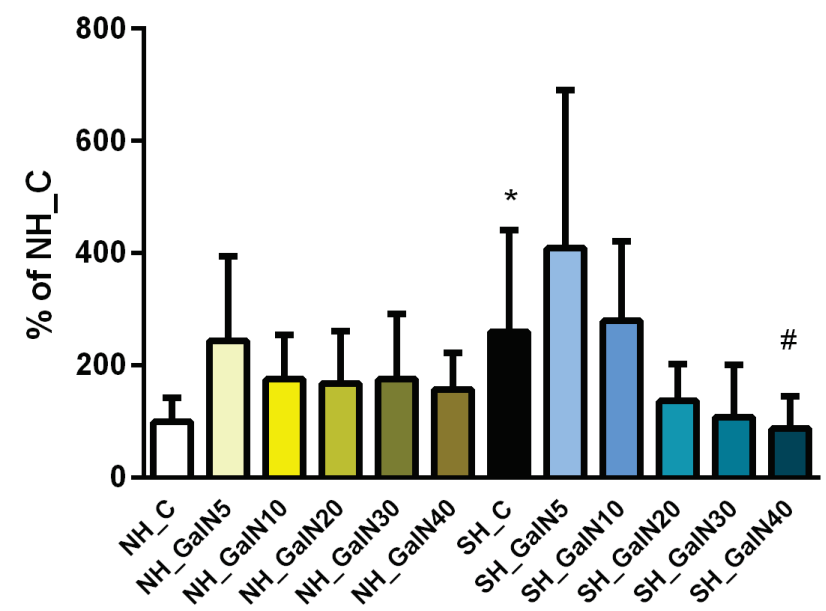

B

\section{Caspase-3 activity in cell lysate}

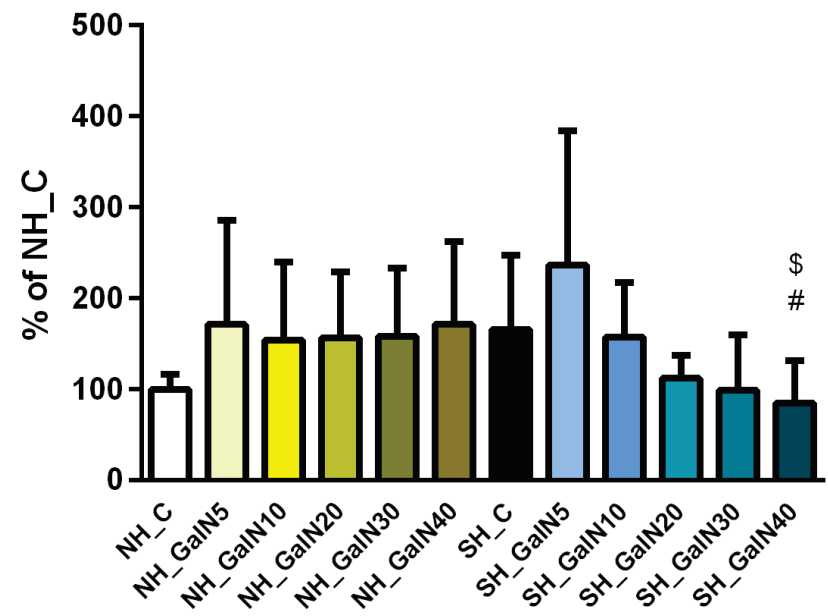

D

\section{Caspase-9 activity in cell lysate}

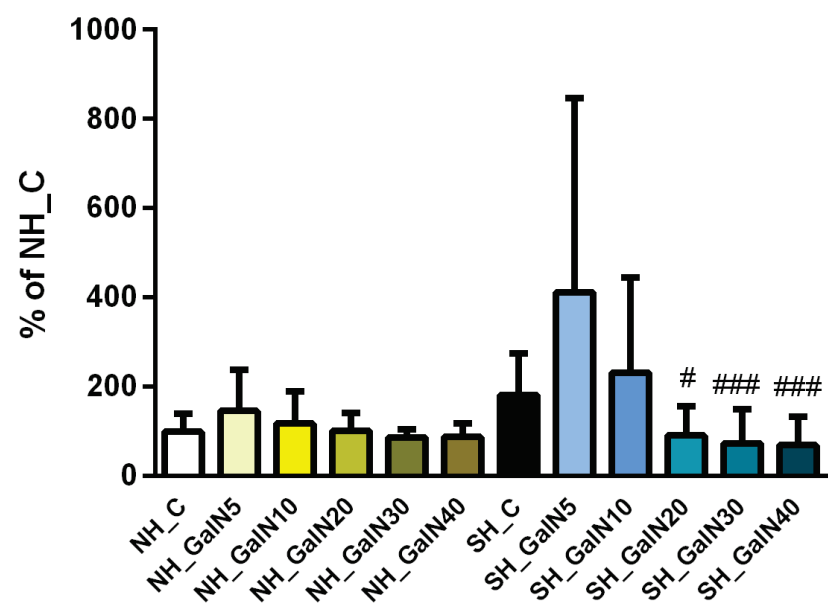

Fig. 1. LDH leakage (A) and activities of caspase $3(\mathbf{B})$, caspase $8(\mathbf{C})$ and caspase $9(\mathbf{D})$ in cell lysate after 24-h incubation of lean and steatotic hepatocytes without (NH_C and $\mathrm{SH} \_\mathrm{C}$, respectively) or with GalN (NH_GalN and SH_GalN, respectively). The values are means \pm SD of 3 (for $A, C, D, n=16-18$ ) or 4 (for $B, n=23-24)$ independent experiments. Data are expressed in \% of $N H \_C(B, C$ and D). $* p<0.05$ and $* * * p<0.001$ vs. NH_C; $\# p<0.05$ and \#\#\# $p<0.001$ vs. SH_C; $\$ p<0.05$ and $\$ \$ \$ p<0.001$ vs. NH_GalN of appropriate concentration of GalN

\section{Discussion}

Recently, several clinical studies showed that patients with NAFLD exert higher risk of drug-induced liver injury than general population (Nguyen et al. 2008, Tarantino et al. 2007, 2009). Also some experimental works confirmed higher susceptibility of fatty hepatocytes to toxic injury in vivo (Aubert et al. 2012, Kucera et al. 2012b, 2011b, Sawant et al. 2006) and in vitro (Kucera et al. 2012a, 2014, Luo et al. 2012). In the present study, we demonstrate that steatotic hepatocytes in primary cultures are more sensitive to the toxic effect of GalN.

D-galactosamine blocks transcription and protein synthesis in the liver via depletion of uridine nucleotides in hepatocytes (Keppler et al. 1970). Both modes of cell death, apoptosis and necrosis, participate on hepatotoxic effect of GalN in vivo (Cuesta et al. 2006, Kuhla et al. 2009). Transcriptional arrest of hepatocytes by GalN sensitizes liver to TNF $\alpha$ which is considered to be one of the main causes of GalN-induced liver injury (Leist et al. 1994). In vivo, sensitization of liver parenchymal cells to TNF $\alpha$ leads to the extensive apoptosis (Leist et al. 1994, Saavedra et al. 1997). 
A

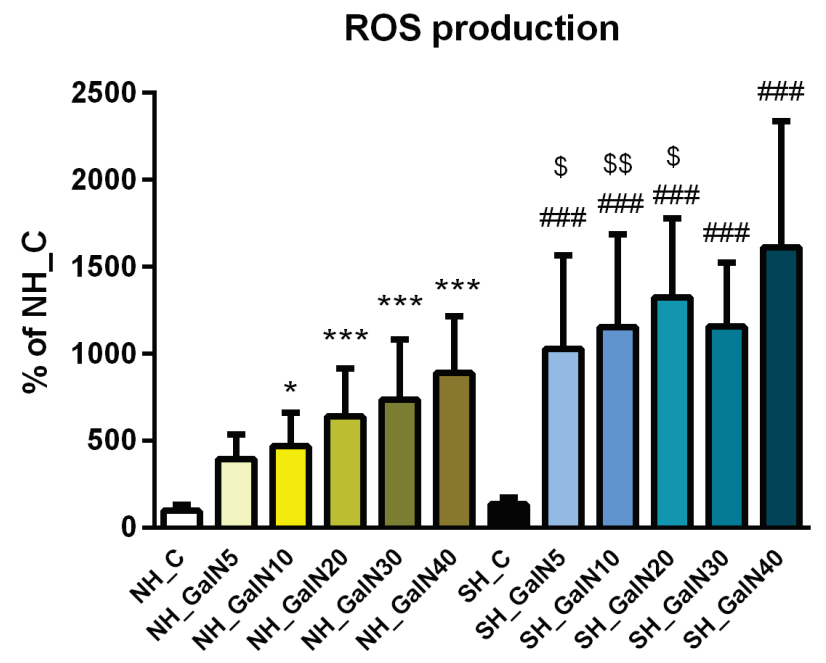

B

\section{Concentration of MDA in cell lysate}

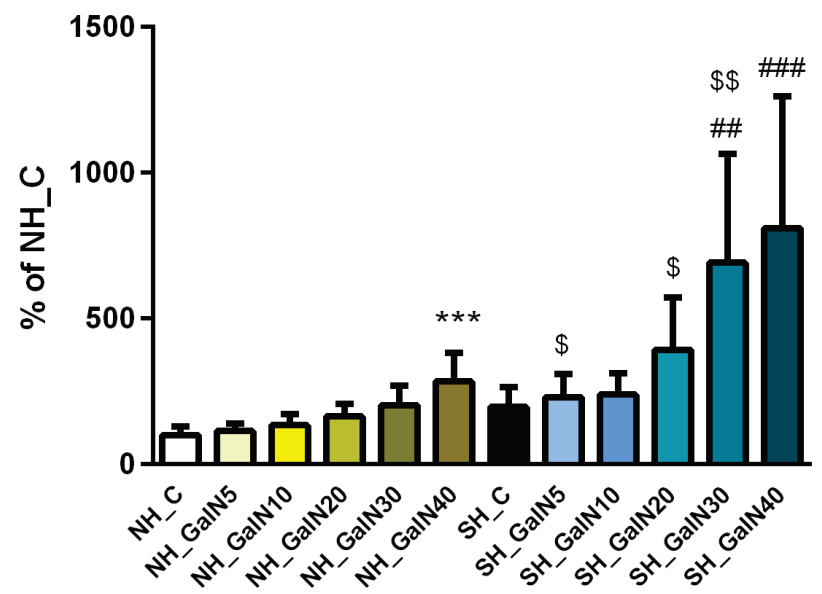

Fig. 2. Production of ROS (A) and concentration of MDA in cell lysate (B) after 24-h incubation of lean and steatotic hepatocytes without (NH_C and SH_C, respectively) or with GalN (NH_GalN and SH_GalN, respectively). The values are means \pm SD of 3 (for $\mathrm{B}, \mathrm{n}=18$ ) or 4 (for $\mathrm{A}, \mathrm{n}=22-24$ ) independent experiments. Data are expressed in $\%$ of NH_C. $* p<0.05$ and $* * * p<0.001$ vs. NH_C; \#\# $p<0.01$ and \#\#\# $p<0.001$ vs. SH_C; $\$ p<0.05$ and $\$ \$ p<0.01$ vs. NH_GalN of appropriate concentration of GalN

Apoptosis was proved to be involved in GalN-induced rat hepatocyte damage in vitro as well (Siendones et al. 2005). Results of this manuscript show that necrosis strongly prevails over apoptosis in our experimental conditions. Intracellular activities of caspase 8 and caspase 9, the initiator caspases of the extrinsic and mitochondrial apoptotic pathways, respectively, showed only a non-significant trend of increased activities in the lowest tested dose of GalN (5 mmol/l) in lean and steatotic hepatocytes. Activity of caspase 3 in cell lysate was not increased in any group incubated with GalN. Our results of caspase 8 and 9 activities correspond to the

\section{Concentration of TNF $\alpha$ in culture medium}

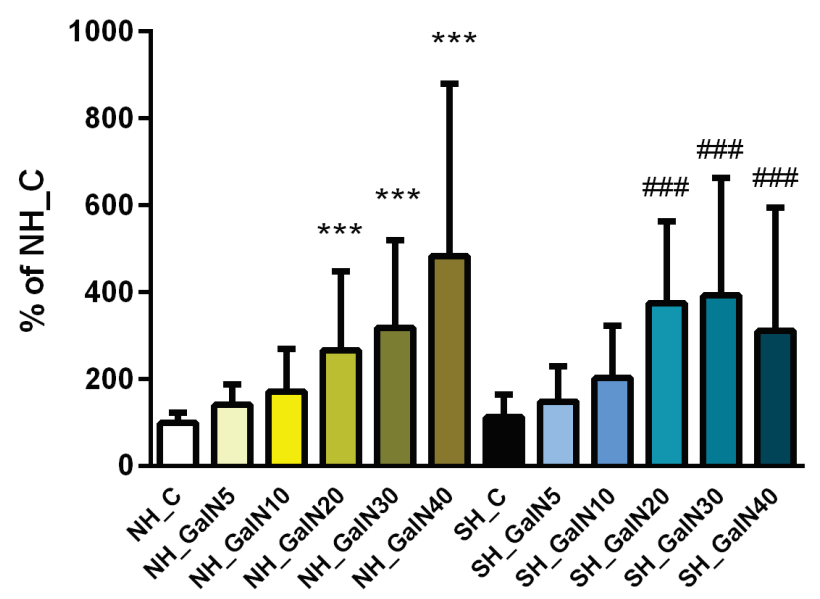

Fig. 3. Concentration of TNFa in culture medium after 24-h incubation of lean and steatotic hepatocytes without (NH_C and SH_C, respectively) or with GalN (NH_GalN and SH_GalN, respectively). The values are means \pm SD of 4 independent experiments $(n=22-24)$. Data are expressed in \% of $\mathrm{NH}_{-} \mathrm{C}$. *** $p<0.001$ vs. NH_C; \#\#\# $p<0.001$ vs. SH_C

\section{Percentage of hepatocytes with energized mitochondria}

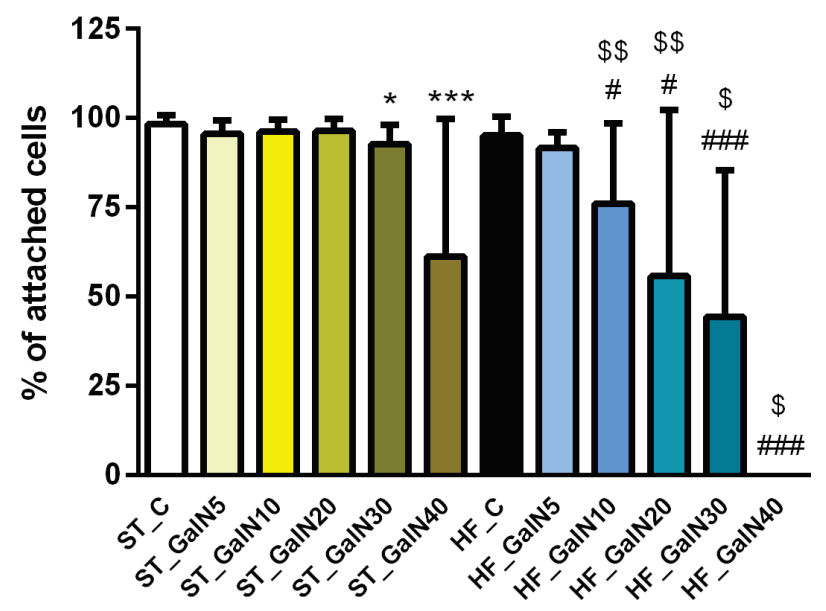

Fig. 4. Percentage of attached hepatocytes containing energized mitochondria after 24-h incubation of lean and steatotic hepatocytes without (NH_C and SH_C, respectively) or with GalN (NH_GalN and SH_GalN, respectively). The values are means \pm SD of 3 independent experiments $(n=18-28)$. $* p<0.05$ and $* * * p<0.001$ vs. NH_C; \# $p<0.05$ and \#\#\# $p<0.001$ vs. SH_C; $\$ p<0.05$ and $\$ \$ p<0.01$ vs. NH_GalN of appropriate concentration of GalN

results of Siendones et al. (2005) who found that these caspases are not activated in GalN toxicity in rat hepatocytes in vitro. As concerns the activity of caspase 3 , we did not observe any activation of this caspase in GalN-induced injury to rat hepatocytes which is in contrast to findings of Siendones et al. (2005). Different 
results of caspase- 3 activation by GalN may be explained by distinct cultivation protocols. Whereas we exposed hepatocytes to GalN immediately after the seeding process, the other group of authors delayed administration of GalN to hepatocytes by $24 \mathrm{~h}$ (Siendones et al. 2005). This surmise is supported by findings of Canova et al. (2008) who found that GalN is more potent to induce hepatocyte apoptosis during 24 to $48 \mathrm{~h}$ incubation than during 0 to $24 \mathrm{~h}$ period.

A

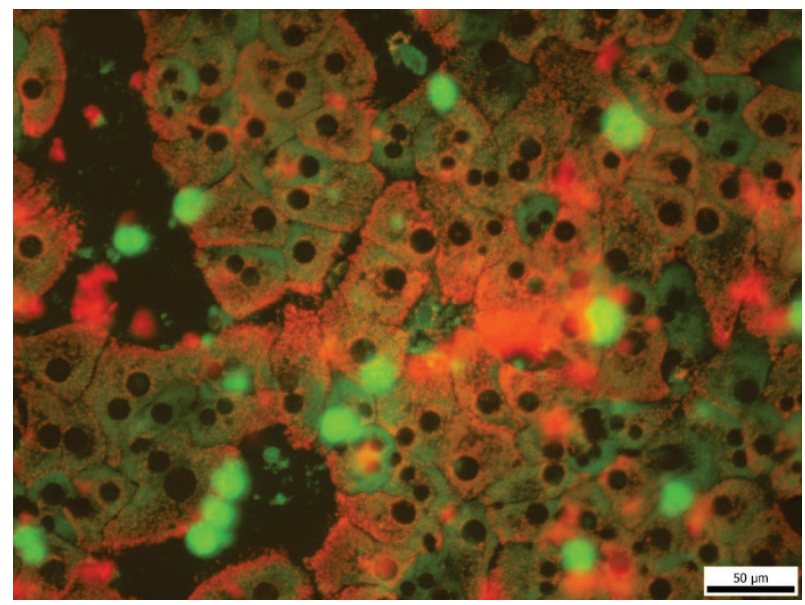

C

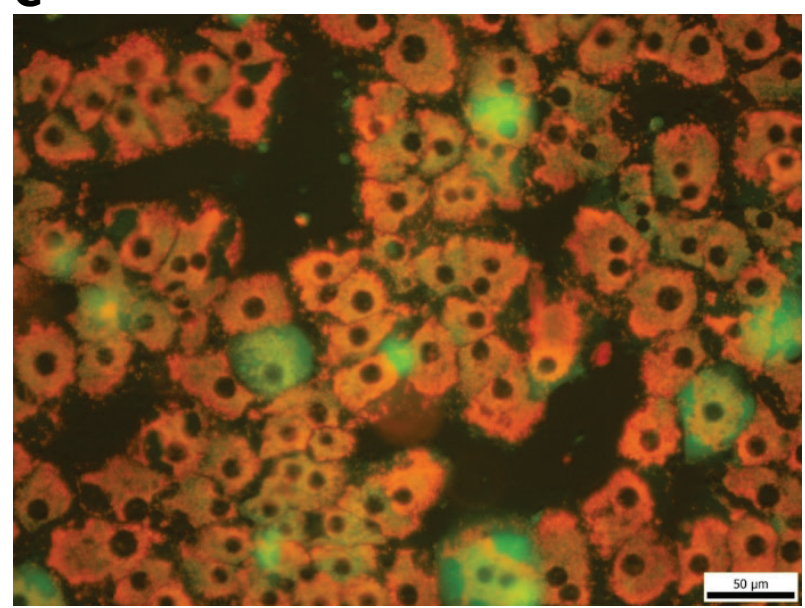

Necrotic death of hepatocytes was monitored by LDH leakage which was significantly increased from $30 \mathrm{mM}$ and $20 \mathrm{mM}$ concentration of GalN in non-steatotic and fatty hepatocytes, respectively. Significantly increased activity of caspase 8 in control fatty hepatocytes when compared to control lean cells together with mentionable trend of elevated activities of caspase 3 and caspase 9 in the same experimental group may indicate that steatotic hepatocytes predispose to apoptosis.

\section{B}

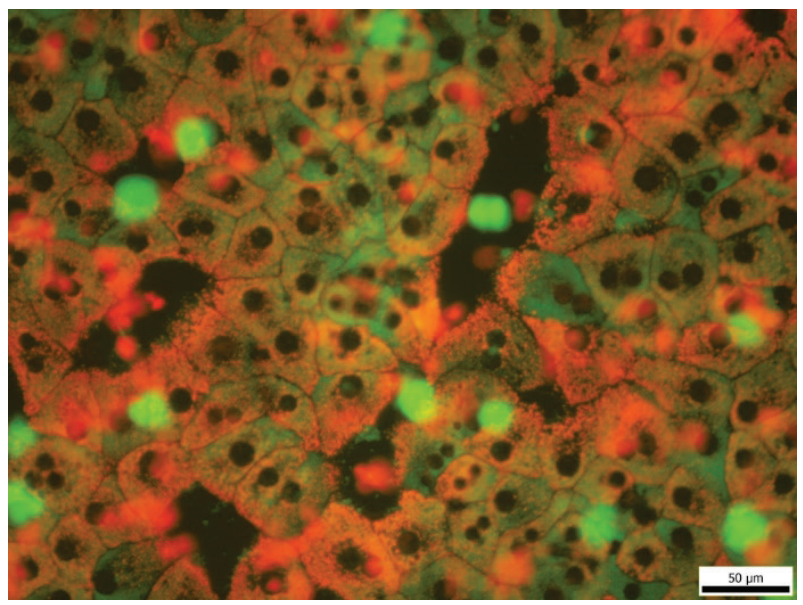

D

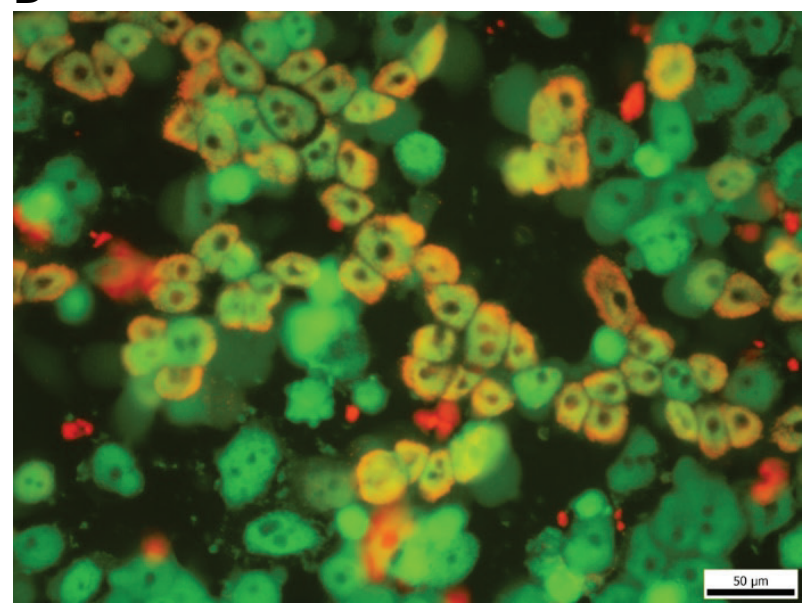

Fig. 5. Visualization of changes in mitochondrial membrane potential using mitochondria-specific fluorescent probe JC-1. Mitochondria with intact membrane potential concentrate JC-1 and exert orange fluorescence whereas de-energized mitochondria emit green fluorescence. Microphotographs of non-steatotic and fatty rat hepatocytes cultured in William's E medium for $24 \mathrm{~h}$ without (A and B, respectively) or with $20 \mathrm{mM}$ GalN (C and D, respectively). Objective magnification 40x, bar $50 \mu \mathrm{m}$

Although the role of TNF $\alpha$ in GalN toxicity in vivo is well known, the importance of TNF $\alpha$ in GalNinduced hepatocyte injury in vitro is not well understood. We did not find any difference in levels of TNFa in culture medium between lean and steatotic control hepatocytes which correlates well with the absence of significant differences in both serum (Kucera et al. 2011b) and hepatic (Kucera et al. 2012b) contents of TNF $\alpha$ between lean rats and animals with simple steatosis in vivo. Exposure of hepatocytes to GalN led to a similar dose-dependent elevation in TNF $\alpha$ concentration in both lean and fatty groups. Despite the significance of TNF $\alpha$ in apoptosis, we did not find noticeable activation of apoptotic mode of cell death in GalN-treated hepatocytes 
as mentioned above.

Oxidative stress and nitrosative stress were reported to contribute significantly to GalN toxicity (McMillan and Jollow 1992, Quintero et al. 2002, Ranchal et al. 2006). Oxidative stress does not seem to be caused primarily by GalN, but it is rather a consequence of metabolic disturbances induced by depletion of uridine nucleotides, inhibition of proteosynthesis and synthesis of glutathione (McMillan and Jollow 1992, Quintero et al. 2002). Our study demonstrates that steatotic hepatocytes are more sensitive to the induction of generation of ROS and lipid peroxidation by GalN. All tested concentrations of GalN increased production of ROS in fatty cells whereas the lowest dose of GalN did not elevate significantly ROS formation in lean hepatocytes. Moreover, GalN (5-20 mmol/l) caused significantly higher generation of ROS in steatotic hepatocytes than in lean cells. We have previously demonstrated that steatotic hepatocytes in vitro exert higher production of ROS, increased lipid peroxidation and lower redox state of glutathione (Kučera et al. 2014). Although we did not observe significant differences in ROS generation and lipid peroxidation between control groups of lean and steatotic hepatocytes in this experiment, ROS production and MDA concentration in cell lysate were almost 1.4-fold and 2-fold higher in control fatty cells than in control non-steatotic hepatocytes. These alterations may predispose steatotic hepatocytes to increased susceptibility to oxidative damage.

Mitochondrial dysfunction and mitochondrial permeability transition (MPT) were reported to participate in hepatotoxicity of GalN in vitro and in vivo (Quintero et al. 2002, Soriano et al. 2004). In this work, we estimated MMP by a fluorescent probe JC-1 and calculated hepatocytes containing energized mitochondria. Fatty cells exposed to GalN were significantly more susceptible to loss of MMP than lean hepatocytes. Higher sensitivity of mitochondria to loss of MMP was previously proven in steatotic hepatocytes exposed to exogenous source of oxidative stress (Kučera et al. 2014). Overproduction of ROS is a known inducer of mitochondrial dysfunction and MPT (Halestrap et al. 2002). MPT in hepatocytes can be also induced by TNF $\alpha$ (Hatano et al. 2000). Whereas we did not observe any differences in TNF $\alpha$ between lean and steatotic hepatocytes exposed to GalN, markers of oxidative stress were significantly higher in steatotic hepatocytes treated with GalN.

In conclusion, this study shows that steatotic hepatocytes are more susceptible to the toxic effect of GalN. This sensitivity may result from higher GalN-induced ROS generation and lipid peroxidation and higher susceptibility of mitochondria to loss of mitochondrial membrane potential in steatotic hepatocytes. In our experimental arrangement, apoptosis does not seem to participate considerably on hepatotoxic action of GalN in either group of hepatocytes. Although it is not possible to simply extrapolate the outcomes from non-human experiments in vivo or in vitro to clinical practice, the results of our study and our previous works (Garnol et al. 2014, Kucera et al. 2011b, 2012a, b, 2014) indicate that simple fatty transformation of hepatocytes is not only benign morphological change but predisposes hepatocytes to enhanced susceptibility to hepatotoxins of different mechanisms of action.

\section{Conflict of Interest}

There is no conflict of interest.

\section{Acknowledgements}

This study was supported by the programme PRVOUK P37/02.

\section{References}

AUBERT J, BEGRICHE K, DELANNOY M, MOREL I, PAJAUD J, RIBAULT C, LEPAGE S, MCGILL MR, LUCAS-CLERC C, TURLIN B, ROBIN MA, JAESCHKE H, FROMENTY B: Differences in early acetaminophen hepatotoxicity between obese ob/ob and db/db mice. J Pharmacol Exp Ther 342: 676-687, 2012.

BECHMANN LP, GIESELER RK, SOWA JP, KAHRAMAN A, ERHARD J, WEDEMEYER I, EMONS B, JOCHUM C, FELDKAMP T, GERKEN G, CANBAY A: Apoptosis is associated with CD36/fatty acid translocase upregulation in non-alcoholic steatohepatitis. Liver Int 30: 850-859, 2010.

BERRY MN, EDWARDS AM, BARRITT GJ: High-yield preparation of isolated hepatocytes from rat liver. In: Isolated Hepatocytes Preparation, Properties and Application. BURDON RH, VAN KNIPPENBERG PH (eds), Elsevier, New York, 1991, pp 15-58. 
BRADFORD MM: A rapid and sensitive method for the quantitation of microgram quantities of protein utilizing the principle of protein-dye binding. Anal Biochem 72: 248-254, 1976.

CANOVA NK, MARTINEK J, KMONICKOVA E, ZIDEK Z, KAMENIKOVA L, FARGHALI H: Modulation of spontaneous and lipopolysaccharide-induced nitric oxide production and apoptosis by d-galactosamine in rat hepatocyte culture: the significance of combinations of different methods. Toxicol Mech Methods 18: 63-74, 2008.

CUESTA E, BOADA J, CALAFELL R, PERALES JC, ROIG T, BERMUDEZ J: Fructose 1,6-bisphosphate prevented endotoxemia, macrophage activation, and liver injury induced by D-galactosamine in rats. Crit Care Med 34: 807-814, 2006.

FELDSTEIN AE, CANBAY A, ANGULO P, TANIAI M, BURGART LJ, LINDOR KD, GORES GJ: Hepatocyte apoptosis and fas expression are prominent features of human nonalcoholic steatohepatitis. Gastroenterology 125: 437-443, 2003.

FROMENTY B: Drug-induced liver injury in obesity. J Hepatol 58: 824-826, 2013.

GARCIA-RUIZ I, RODRIGUEZ-JUAN C, DIAZ-SANJUAN T, DEL HOYO P, COLINA F, MUNOZ-YAGUE T, SOLIS-HERRUZO JA: Uric acid and anti-TNF antibody improve mitochondrial dysfunction in ob/ob mice. Hepatology 44: 581-591, 2006.

GARNOL T, ENDLICHER R, KUCERA O, DRAHOTA Z, CERVINKOVA Z: Impairment of mitochondrial function of rat hepatocytes by high fat diet and oxidative stress. Physiol Res 63: 271-274, 2014.

HALESTRAP AP, MCSTAY GP, CLARKE SJ: The permeability transition pore complex: another view. Biochimie 84: 153-166, 2002.

HATANO E, BRADHAM CA, STARK A, IIMURO Y, LEMASTERS JJ, BRENNER DA: The mitochondrial permeability transition augments Fas-induced apoptosis in mouse hepatocytes. J Biol Chem 275: 11814-11823, 2000.

KEPPLER DO, RUDIGIER JF, BISCHOFF E, DECKER KF: The trapping of uridine phosphates by D-galactosamine. D-glucosamine, and 2-deoxy-D-galactose. A study on the mechanism of galactosamine hepatitis. Eur $J$ Biochem 17: 246-253, 1970.

KLEINER DE, BRUNT EM, VAN NATTA M, BEHLING C, CONTOS MJ, CUMMINGS OW, FERRELL LD, LIU YC, TORBENSON MS, UNALP-ARIDA A, YEH M, MCCULLOUGH AJ, SANYAL AJ; NONALCOHOLIC STEATOHEPATITIS CLINICAL RESEARCH NETWORK: Design and validation of a histological scoring system for nonalcoholic fatty liver disease. Hepatology 41: 1313-1321, 2005.

KUCERA O, GARNOL T, LOTKOVA H, STANKOVA P, MAZUROVA Y, HROCH M, BOLEHOVSKA R, ROUSAR T, CERVINKOVA Z: The effect of rat strain, diet composition and feeding period on the development of a nutritional model of non-alcoholic fatty liver disease in rats. Physiol Res 60: 317-328, $2011 \mathrm{a}$.

KUCERA O, LOTKOVA H, STANKOVA P, PODHOLA M, ROUSAR T, MEZERA V, CERVINKOVA Z: Is rat liver affected by non-alcoholic steatosis more susceptible to the acute toxic effect of thioacetamide? Int J Exp Pathol 92: 281-289, 2011 b.

KUCERA O, AL-DURY S, LOTKOVA H, ROUSAR T, RYCHTRMOC D, CERVINKOVA Z: Steatotic rat hepatocytes in primary culture are more susceptible to the acute toxic effect of acetaminophen. Physiol Res $\mathbf{6 1}$ (Suppl 2): S93-S101, 2012a.

KUCERA O, ROUSAR T, STANKOVA P, HANACKOVA L, LOTKOVA H, PODHOLA M, CERVINKOVA Z: Susceptibility of rat non-alcoholic fatty liver to the acute toxic effect of acetaminophen. $J$ Gastroenterol Hepatol 27: 323-330, 2012b.

KUČERA O, ENDLICHER R, ROUŠAR T, LOTKOVÁ H, GARNOL T, DRAHOTA Z, ČERVINKOVÁ Z: The effect of tert-butyl hydroperoxide-induced oxidative stress on lean and steatotic rat hepatocytes in vitro. Oxid Med Cell Longev 2014: Article ID 752506, 2014.

KUHLA A, EIPEL C, ABSHAGEN K, SIEBERT N, MENGER MD, VOLLMAR B: Role of the perforin/granzyme cell death pathway in D-Gal/LPS-induced inflammatory liver injury. Am J Physiol Gastrointest Liver Physiol 296: G1069-G1076, 2009. 
LEHMANN V, FREUDENBERG MA, GALANOS C: Lethal toxicity of lipopolysaccharide and tumor necrosis factor in normal and D-galactosamine-treated mice. $J$ Exp Med 165: 657-663, 1987.

LEIST M, GANTNER F, BOHLINGER I, GERMANN PG, TIEGS G, WENDEL A: Murine hepatocyte apoptosis induced in vitro and in vivo by TNF-alpha requires transcriptional arrest. J Immunol 153: 1778-1788, 1994.

LUO Y, RANA P, WILL Y: Palmitate increases the susceptibility of cells to drug-induced toxicity: an in vitro method to identify drugs with potential contraindications in patients with metabolic disease. Toxicol Sci 129: 346-362, 2012.

MCMILLAN JM, JOLLOW DJ: Galactosamine hepatotoxicity: effect of galactosamine on glutathione resynthesis in rat primary hepatocyte cultures. Toxicol Appl Pharmacol 115: 234-240, 1992.

MERRELL MD, CHERRINGTON NJ: Drug metabolism alterations in nonalcoholic fatty liver disease. Drug Metab Rev 43: 317-334, 2011.

NGUYEN GC, SAM J, THULUVATH PJ: Hepatitis $\mathrm{C}$ is a predictor of acute liver injury among hospitalizations for acetaminophen overdose in the United States: a nationwide analysis. Hepatology 48: 1336-1341, 2008.

OHKAWA H, OHISHI N, YAGI K: Assay for lipid peroxides in animal tissues by thiobarbituric acid reaction. Anal Biochem 95: 351-358, 1979.

PEREZ-CARRERAS M, DEL HOYO P, MARTIN MA, RUBIO JC, MARTIN A, CASTELLANO G, COLINA F, ARENAS J, SOLIS-HERRUZO JA: Defective hepatic mitochondrial respiratory chain in patients with nonalcoholic steatohepatitis. Hepatology 38: 999-1007, 2003.

QUINTERO A, PEDRAZA CA, SIENDONES E, KAMAL ELSAID AM, COLELL A, GARCIA-RUIZ C, MONTERO JL, DE LA MATA M, FERNANDEZ-CHECA JC, MINO G, MUNTANE J: PGE1 protection against apoptosis induced by D-galactosamine is not related to the modulation of intracellular free radical production in primary culture of rat hepatocytes. Free Radic Res 36: 345-355, 2002.

RANCHAL I, GONZALEZ R, LOPEZ-SANCHEZ LM, BARRERA P, LOPEZ-CILLERO P, SERRANO J, BERNARDOS A, DE LA MATA M, RODRIGUEZ-ARIZA A, MUNTANE J: The differential effect of PGE(1) on d-galactosamine-induced nitrosative stress and cell death in primary culture of human hepatocytes. Prostaglandins Other Lipid Mediat 79: 245-259, 2006.

SAAVEDRA JE, BILLIAR TR, WILLIAMS DL, KIM YM, WATKINS SC, KEEFER LK: Targeting nitric oxide (NO) delivery in vivo. Design of a liver-selective NO donor prodrug that blocks tumor necrosis factor-alpha-induced apoptosis and toxicity in the liver. J Med Chem 40: 1947-1954, 1997.

SAWANT SP, DNYANMOTE AV, WARBRITTON A, LATENDRESSE JR, MEHENDALE HM: Type 2 diabetic rats are sensitive to thioacetamide hepatotoxicity. Toxicol Appl Pharmacol 211: 221-232, 2006.

SIENDONES E, JIMENEZ-GOMEZ Y, MONTERO JL, GOMEZ-DIAZ C, VILLALBA JM, MUNTANE J: PGE1 abolishes the mitochondrial-independent cell death pathway induced by D-galactosamine in primary culture of rat hepatocytes. J Gastroenterol Hepatol 20: 108-116, 2005.

SORIANO ME, NICOLOSI L, BERNARDI P: Desensitization of the permeability transition pore by cyclosporin a prevents activation of the mitochondrial apoptotic pathway and liver damage by tumor necrosis factor-alpha. J Biol Chem 279: 36803-36808, 2004.

STACHLEWITZ RF, SEABRA V, BRADFORD B, BRADHAM CA, RUSYN I, GERMOLEC D, THURMAN RG: Glycine and uridine prevent D-galactosamine hepatotoxicity in the rat: role of Kupffer cells. Hepatology 29: 737-745, 1999.

TARANTINO G, CONCA P, BASILE V, GENTILE A, CAPONE D, POLICHETTI G, LEO E: A prospective study of acute drug-induced liver injury in patients suffering from non-alcoholic fatty liver disease. Hepatol Res 37: 410-415, 2007.

TARANTINO G, Di MINNO MN, CAPONE D: Drug-induced liver injury: is it somehow foreseeable? World $J$ Gastroenterol 15: 2817-2833, 2009.

TILG H: The role of cytokines in non-alcoholic fatty liver disease. Dig Dis 28: 179-185, 2010.

VERNON G, BARANOVA A, YOUNOSSI ZM: Systematic review: the epidemiology and natural history of nonalcoholic fatty liver disease and non-alcoholic steatohepatitis in adults. Aliment Pharmacol Ther 34: 274-285, 2011. 
VIDELA LA, RODRIGO R, ORELLANA M, FERNANDEZ V, TAPIA G, QUINONES L, VARELA N, CONTRERAS J, LAZARTE R, CSENDES A, ROJAS J, MALUENDA F, BURDILES P, DIAZ JC, SMOK G, THIELEMANN L, PONIACHIK J: Oxidative stress-related parameters in the liver of non-alcoholic fatty liver disease patients. Clin Sci (Lond) 106: 261-268, 2004. 